\title{
BOUNDS IN SPACES OF MORREY UNDER CHICCO TYPE CONDITIONS
}

\author{
A. CAnAle
}

Abstract. In the present paper we consider Morrey spaces in unbounded domains and study elliptic equations in nondivergence form with discontinuous coefficients when the class of discontinuities is of Chicco type. In particular we state some local and non local a priori bounds for solutions of Dirichlet problem and study the dependence of the constants in the estimates. The idea is to approximate the principal coefficients by functions with derivatives which belong locally to the space $L^{s}, 2<s \leqslant n$, while the coefficients of lower terms in the differential operator belong to Morrey spaces. Our results are based on embedding theorems which allow us to require a summability lower than $n$ for the coefficients of the operator $L$.

Mathematics subject classification (2000): 35J25, $46 \mathrm{E} 35$. condition

Keywords and phrases: elliptic equations, embedding theorems, a priori bounds, Morrey spaces, Chicco

\section{REFERENCES}

[1] R. A. ADAMS, Sobolev spaces, Academic Press, 1975.

[2] A. Alvino, G. TROmbetti, Second order elliptic equations whose coefficients have their first derivatives weakly- $L^{n}$, Ann. Mat. Pura Appl. 138 (1984), 331-340.

[3] A. Canale, A priori bounds in weighted spaces, J. Math. Anal. Appl. Vol. 287 (4) (2003), 101-117.

[4] A. CANALE, Existence and uniqueness results in weighted spaces, J. Concr. Appl. Math. Vol. 1 (4) (2003), 307-323.

[5] A. CANALE, On some results in weighted spaces under Cordes type conditions, J. Interdiscip. Math., Vol. 10 (2)(2007), 245-261.

[6] A. CANALE, On some results in weighted spaces under Chicco type conditions, Int. J. Pure Appl. Math., Vol. 31 (2) (2006), 185-202.

[7] A. Canale, Bounds in spaces of Morrey under Cordes type conditions, J. Appl. Funct. Anal., Vol. 3 (1) (2008), 11-32.

[8] A. Canale, L. CASo, M. Transirico, Second order elliptic equations with discontinuous coefficients in irregular domains Rend. Accad. Naz. Sci. XL Mem. Mat., 24 (2000), 63-79.

[9] A. Canale, P. Di Gironimo, A. Vitolo, Functions with derivatives in spaces of Morrey type and elliptic equations in unbounded domains, Studia Math. (3) 128 (1998), 199-218.

[10] A. CANALE, M. LONGOBARDI, G. MANZO, Second order elliptic equations with discontinuous coefficients in unbounded domains, Rend. Accad. Naz. Sci. XL Mem. Mat. 18 (1994), 41-56.

[11] A. Canale, M. Longobardi, G. Manzo, Existence and uniqueness results for second order elliptic equations in unbounded domains, Rend. Accad. Naz. Sci. XL Mem. Mat. 18 (1994), 171-187.

[12] L. CASO, M. TRANSIRICO, The Dirichlet problem for second order elliptic equations with singular data, Acta Math. Hungar. 76 (1997), 1-16.

[13] F. Chiarenza, M. Franciosi, A generalization of a theorem by C.Miranda, Ann. Mat. Pura Appl. 161 (1992), 285-297.

[14] F. Chiarenza, M. Frasca, A remark on a paper by C. Miranda, Proc. Amer. Math. Soc. 108 (1990), 407-409.

[15] F. Chiarenza, M. Frasca, P. LONGO, Interior $W^{2, p}$ estimates for non divergence elliptic equations with discontinuous coefficients, Ricerche Mat. 40 (1991), 149-168. 
[16] F. Chiarenza, M. FrasCa, P. Longo, $W^{2, p}$-solvability of the Dirichlet problem for nondivergence elliptic equations with VMO coefficients, Trans. Amer. Math. Soc. 336 (1993), 841-853.

[17] M. CHICCO, Dirichlet problem for a class of linear second order elliptic partial differential equations with discontinuous coefficients, Ann. Mat. Pura Appl. 92 (1972), 13-22.

[18] M. CHICCO, Principio di massimo per soluzioni di equazioni ellittiche del secondo ordine di tipo Cordes, Ann. Mat. Pura Appl. 100 (1974), 239-258.

[19] M. CHICCO, Osservazioni sulla risolubilità del problema di Dirichlet per una classe di equazioni ellittiche a coefficienti discontinui, Rend. Sem. Mat. Univ. Padova 66 (1982), 137-141.

[20] M. CHICCO, Su un classe di equazioni ellittiche del secondo ordine in forma non variazionale, Boll. Unione Mat. Ital. 4-A (1985), 479-486.

[21] C. FefFERman, The uncertainty principle, Bull. Amer. Math. Soc. 9 (1983), 129-206.

[22] M. FRANCIOSI, N. FusCO, $W^{2, p}$ regularity for the solutions of elliptic non divergence form equations with rough coefficients, Ricerche Mat., 38 (1989), 93-106.

[23] C. MiRANDA, Sulle equazioni ellittiche di tipo non variazionale a coefficienti discontinui, Ann. Mat. Pura Appl. 63 (1963), 353-386.

[24] G. TALENTI, Sopra una classe di equazioni ellittiche a coefficienti misurabili, Ann. Mat. Pura Appl. 69 (1965), 285-304.

[25] M. Transirico, M. Troisi, Equazioni ellittiche del secondo ordine di tipo non variazionale in aperti non limitati, Ann. Mat. Pura Appl. 152 (1988), 209-226.

[26] M. TRAnsirico, M. TRoisi, A. Vitolo, Spaces of Morrey type and elliptic equations in divergence form on unbounded domains, Boll. Unione Mat. Ital. 9 (1995), 153-174.

[27] G. VIOLA, Sulle equazioni ellittiche del secondo ordine a coefficienti non regolari, Rend. Mat. 4 (1984), 617-632. 